\title{
Temporal Masking in Electric Hearing
}

\author{
Fan-Gang Zeng, ${ }^{1}$ Hongbin Chen, ${ }^{1}$ and Shilong Han ${ }^{2}$ \\ ${ }^{1}$ Departments of Anatomy and Neurobiology, Biomedical Engineering, Cognitive Sciences and Otolaryngology - Head and \\ Neck Surgery, University of California, Irvine, CA 92697, USA \\ ${ }^{2}$ Department of Otolaryngology - Head and Neck Surgery, Jiujiang University Hospital, Jiujiang 332000, China
}

Received: 25 March 2005; Accepted: 26 August 2005; Online publication: 29 October 2005

\begin{abstract}
Temporal masking can be defined as the detection threshold of a brief signal as a function of the signal delay in a relatively long masker. The temporal masking pattern in normal acoustic hearing reveals temporal edge enhancement in which the signal detection threshold is greater near the masker onset than in the steady-state portion. Both peripheral and central mechanisms appear to underlie temporal edge enhancement, but their relative contributions remain elusive. Cochlear implants bypass cochlear mechanical processing and stimulate the auditory nerve directly, thereby providing a unique opportunity to separate the peripheral mechanisms from the central mechanisms. Here, we systematically measured temporal masking in electric hearing by examining whether a brief signal was harder to detect at the onset than in the steady-state portion of a long masker (the "overshoot" effect). The signal and the masker were presented (1) either to the same electrode or to different electrodes, (2) at the same stimulation or different rates, and (3) in a simultaneous or an interleaved fashion. A consistent pattern of results was observed, depending on the stimulus configuration between the signal and the masker. Simultaneous stimulation at the same rate and with the same electrode produced no difference in sensitivity between the onset and the steady-state conditions, but interleaved stimulation at different rates or with different electrodes produced a significant difference. Unlike acoustic hearing, high masker levels produced an overshoot effect, and low
\end{abstract}

Correspondence to: Fan-Gang Zeng - Hearing and Speech Research Laboratory, 364 Med Surge II • University of California • Irvine, CA 92697-1275, USA. Telephone: +1-949-8241539; fax: +1-9498245907; email: fzeng@uci.edu masker levels produced an undershoot effect. Although the present results are consistent with the "on-frequency vs. off-frequency" hypothesis for the overshoot effect, results also suggest a central "same vs. different" mechanism underlying temporal masking. These results have practical implications for improving cochlear implant design.

Keywords: temporal masking, temporal edge enhancement, overshoot, undershoot, electric hearing, cochlear implant, electrode interaction

\section{INTRODUCTION}

The enhancement of changes in a stimulus is a major mechanism in sensory processing that serves many important functions in nature. For example, the eye uses photochemical adaptation and the ear uses cochlear compression to maintain sharp sensitivity over a large dynamic range (Dowling 1967; Johnstone and Yates 1974; Rhode and Robles 1974; Viemeister 1983). The eye uses lateral inhibition at the neural level to enhance spatial contrast (Dowling 1967; Hartline 1969; Hartline et al. 1956), whereas the ear uses two-tone suppression to enhance spectral peaks (Abbas and Sachs 1976; Sachs and Kiang 1968). The brain has employed sophisticated mechanisms, such as differential receptive fields, to enhance temporal onset and offset or edge detection in both audition and vision (Fishbach et al. 2001; Formby et al. 2000; Marr and Hildreth 1980; Rodieck and Stone 1965). This enhancement of changes in intensity, frequency, and time allows sensory systems to extract critical cues in a complex environment for object detection, formation, and segregation (Bregman 1994; Yost 1991). This study focuses on temporal edge enhance- 
ment, namely, the overshoot effect. Although the overshoot effect has been extensively studied, its underlying mechanisms remain elusive.

The overshoot was first documented four decades ago (Elliott 1965; Zwicker 1965). It refers to the decreased sensitivity to a brief signal near the onset of a masker relative to the sensitivity in a steady-state portion of the masker. For a brief pure-tone signal in long-duration white noise, the difference in the tone detection threshold between the onset and steadystate conditions can be as large as $20 \mathrm{~dB}$ (the size of the overshoot effect depends highly on individuals). Subsequent studies have found many factors that influence the overshoot effect.

First, the overshoot effect is level-dependent (Bacon 1990; Carlyon and White 1992; Summers 2001). Presenting a $10-\mathrm{ms}, 4000-\mathrm{Hz}$ pure tone at either $1-\mathrm{ms}$ or 195-ms delay from the onset of a 400-ms noise, Bacon (1990) found that the overshoot was greatest (10-15 dB) at moderate noise levels (10-30 dB SPL spectrum levels) but negligible at low and high noise levels. Carlyon and White (1992) found that this level-dependent overshoot effect also appeared to depend on signal frequency. Only the high-frequency $(6500 \mathrm{~Hz})$ signal produced a nonmonotonic overshoot effect. Using harmonic stimuli with either the positive or negative Schroeder-phase as the masker, Summers (2001) found that a 60-dB SPL signal produced significantly greater overshoot than an 80-dB SPL signal.

Second, the overshoot effect depends on spectral separation between the signal and the masker (Bacon and Smith 1991; Bacon and Viemeister 1985; Carlyon and White 1992; McFadden 1989; Wright 1995; Zwicker 1965). The degree of overshoot is critically dependent upon whether the masker produces excitation in regions not excited by the signal. For example, the overshoot increased from a few decibels when the noise bandwidth was varied from 0.0 to 0.4 times the signal frequency to $10-20 \mathrm{~dB}$ when a broadband noise was used or when the noise frequency was remote from, particularly higher than, the signal frequency.

Third, there is ample evidence that overshoot is sensitive to cochlear nonlinearity or compression (Strickland 2001; Summers 2001). For example, Summers (2001) found that a positive Schroederphase stimulus produced greater overshoot than a negative Schroeder-phase stimulus. Summers suggested that this difference was attributable to the more modulated cochlear response to the positive Schroeder-phase stimuli. Cochlear damage tends to make the cochlea more linear, thus reducing (Carlyon and Sloan 1987) or removing the overshoot effect in hearing-impaired subjects (Bacon and Takahashi 1992). Temporary loss of cochlear nonlin- earity as a result of temporary threshold shift (Champlin and McFadden 1989) or aspirin consumption also reduces or removes the overshoot effect in normal-hearing subjects (McFadden and Champlin 1990).

These results led investigators to suggest that there are at least two distinct mechanisms underlying the overshoot effect. An on-frequency mechanism might be related to the auditory nerve adaptation (Chatterjee and Smith 1993; Smith 1979) or auditory filters that narrow with time (Wright 1995). An off-frequency mechanism might be centrally mediated but could certainly be influenced by cochlear nonlinearity (Carlyon and White 1992; McFadden 1988; Strickland 2001). There are also two possible central mechanisms contributing to the overshoot effect. One central mechanism is mediated via the olivocochlear efferent fibers. This central mechanism is hypothesized based on the fact that patients who have sectioned efferents show a greatly reduced overshoot effect (Zeng et al. 2000b) and, additionally, a contralateral forward masker influences the overshoot effect (Bacon and Liu 2000). The other central mechanism may be mediated by temporal changes in attention filters (Wright and Dai 1994a,b). The olivocochlear efferent fibers may also be involved because the efferent-sectioned subjects show greatly widened auditory attention filters (Scharf et al. 1994, 1997).

Cochlear implants bypass the mechanical processing in the cochlea and stimulate the auditory nerve directly via electric currents, thus providing a unique opportunity to separate the peripheral mechanisms (cochlear nonlinearity) from the central mechanisms. Several groups have recently measured the overshoot effect in cochlear-implant subjects. Smith et al. (2003) used a 500-Hz, 20-ms probe presented at various signal delays from the onset of a $500-\mathrm{Hz}$, $300-\mathrm{ms}$ masker. The probe and the masker were interleaved in time and delivered to the same or different electrode pairs in three Nucleus-22 users. They found a consistent 1.5-2.0 dB overshoot effect under all conditions. One should note that the use of a $500-\mathrm{Hz}$ signal typically produced a small overshoot effect in acoustic hearing (Wier et al. 1977). Donaldson (personal communication) also measured overshoot in two Clarion users and found similar degrees of overshoot. Using an indirect measure of the overshoot effect, Wojtczak et al. (2003) measured intensity discrimination under gated (similar to signal at the onset of the noise) and fringe (similar to signal in the steady-state portion of the noise) configurations in both Nucleus-22 $(800-\mathrm{Hz}$ pulse train in bipolar mode) and Clarion-v1.2 (1000-Hz pulses in monopolar mode) users. They found a level-dependent difference between the gated and 
fringe conditions in the Clarion users but not in the Nucleus users. At high levels, intensity discrimination in Clarion users was worse with the gated condition than the fringe condition, suggesting an overshoot effect. The reason for the different results between Clarion and Nucleus users is unclear.

The present study systematically investigated the overshoot effect in cochlear-implant subjects. A wide range of stimulus configurations was used to determine the conditions that were necessary and sufficient to produce the overshoot effect. The main experiment used the Nucleus device, but preliminary data were also obtained in two implant subjects using the Ineraid device, which used analog stimulation (sinusoid and noise). The present study served two additional purposes. First, the present study could shed light on the physiological and cognitive mechanisms underlying the overshoot effect. Second, it could help with the design of cochlear implants that restore normal dynamic processing properties in electric hearing.

\section{METHODS}

\section{Subjects}

Six cochlear-implant subjects, including two Ineraid and four Nucleus users, participated in this study. Table 1 shows detailed demographic and audiological information. The data from the two Ineraid subjects were preliminary but motivated the systematic study conducted in Nucleus users. Because the FDA has not approved the percutaneous plug in the Ineraid device, many Ineraid patients have chosen to be explanted and then reimplanted with another FDA-approved device. However, the Ineraid device provided direct and accurate control of the electric stimulus delivered to the electrodes, without bandwidth and other hardware limitations imposed by transcutaneous transmission in modern implants, e.g., stimulating the electrode with analog white noise. For this reason, the preliminary data from the two Ineraid users are included in this report.
Both Institutional Review Board (IRB) approval and written informed consent were obtained from all subjects before data collection.

\section{Stimuli}

In Ineraid subject \#1, sensitivity was evaluated as a function of signal delay for a 9-ms, $200-\mathrm{Hz}$ sinusoidal signal presented in the presence of a $200-\mathrm{ms}, 2000-\mathrm{Hz}$ sinusoidal masker. Both the signal and the masker had 3-ms cosine-squared onset and offset ramps. The signal delay was varied from -150 to $350 \mathrm{~ms}$, with the signal delays from -150 to $0 \mathrm{~ms}$ representing backward masking, 0 to $200 \mathrm{~ms}$ representing simultaneous masking, and 200 to $350 \mathrm{~ms}$ representing forward masking. All stimuli were delivered to the most apical electrode in the monopolar mode. The masker level was fixed at $100 \mu \mathrm{A}$, producing a comfortably loud sensation. The signal threshold was $61 \mu \mathrm{A}$ in the absence of the masker. The result will be presented as a sensation level in $\mathrm{dB}$, in reference to this threshold value.

In Ineraid subject \#2, the growth of the masking function was obtained for detection of a 9-ms, 1000$\mathrm{Hz}$ sinusoidal signal as a function of the masker level. The masker was either a $500-\mathrm{ms}, 1000-\mathrm{Hz}$ sinusoid or $500-\mathrm{ms}$ white noise. The signal delay was 3 or $300 \mathrm{~ms}$ from the onset of the masker. All stimuli had 3-ms ramps and were delivered to the most apical electrode in monopolar mode. The electric dynamic range, from threshold to maximal comfort loudness, was $20-200 \mu \mathrm{A}$ for the sinusoidal masker and 18-398 $\mu \mathrm{A}$ for the noise masker. The masker level was varied from threshold to comfortable loudness level. The 9-ms signal threshold was $24 \mu \mathrm{A}$ in the absence of the masker.

Four conditions were tested with the Nucleus implant subjects to systematically investigate various mechanisms underlying the overshoot effect. Figure 1 shows the detailed configuration of these four stimulus conditions. The masker was always a 400-ms biphasic pulse train (100 $\mu \mathrm{s} /$ phase). To clearly show the relationship between the masker and the 10-ms,

TABLE 1

\begin{tabular}{|c|c|c|c|c|c|c|c|c|}
\hline \multicolumn{9}{|c|}{ Cochlear implant subject information } \\
\hline Subject & Gender & Age (years) & Etiology & Implant & Cl use & Strategy & Vowel & Consonant \\
\hline $\ln 1$ & M & 65 & Noise & Ineraid & 23 & CA & 63 & 57 \\
\hline $\ln 2$ & $\mathrm{~F}$ & 35 & Meningitis & Ineraid & 11 & CA & 33 & 38 \\
\hline S1 & $\mathrm{F}$ & 71 & Fever & N24 & 4 & SPEAK & 51 & 54 \\
\hline S2 & $\mathrm{F}$ & 70 & Virus & N24 & 7 & SPEAK & 64 & 70 \\
\hline S3 & M & 64 & Unknown & N22 & 14 & SPEAK & 75 & 43 \\
\hline S4 & $M$ & 45 & Trauma & N22 & 10 & SPEAK & 86 & 79 \\
\hline
\end{tabular}

$\mathrm{N} 24=$ Nucleus $24, \mathrm{~N} 22=$ Nucleus $22, \mathrm{Cl}$ use $=$ cochlear implant usage in years, CA = compressed analog, SPEAK $=$ spectral PEAK, Vowel $=12 \mathrm{~h} / \mathrm{V} / \mathrm{d}-\mathrm{vowel}$ recognition in percent correct; Consonant $=16 \mathrm{a} / \mathrm{C} / \mathrm{a}$-consonant recognition in percent correct, $\mathrm{NA}=$ not available. 

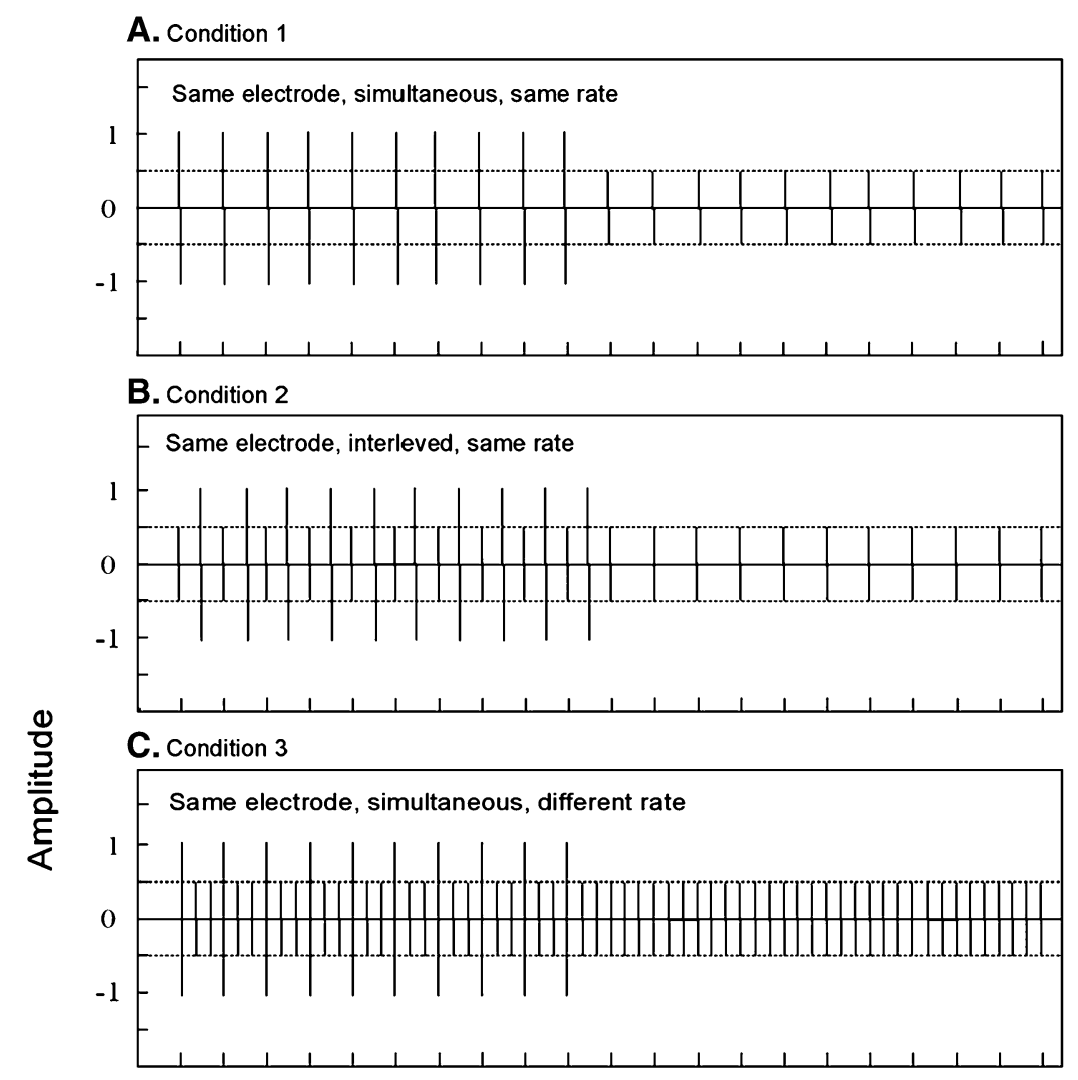

D. Condition 4

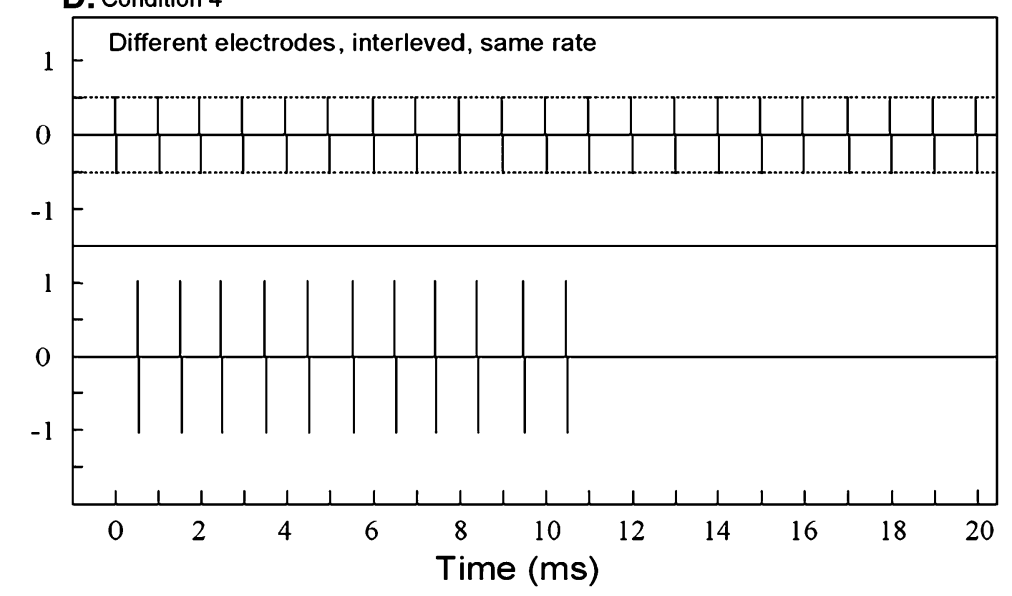

FIG. 1. Stimulus configuration for four experimental conditions in the Nucleus implant subjects. The signal was a $10-\mathrm{ms}, 1000-\mathrm{Hz}$ biphasic pulse train. The masker was a $400-\mathrm{ms}$ biphasic pulse train (only the first 20-ms train was shown here). (A) The signal and the masker were delivered to the same electrode at the same $1000-\mathrm{Hz}$ rate simultaneously (Condition 1). (B) The signal and the masker were delivered to the same electrode at the same $1000-\mathrm{Hz}$ rate but

$100-\mu \mathrm{sec} /$ phase, $1000-\mathrm{Hz}$ signal (or probe), Figure 1 depicts only the onset signal delay (0-ms delay) condition and the first $20 \mathrm{~ms}$ of the masker. The signal is shown as the pulse train with the higher amplitude, whereas the masker is shown as the pulse train with the lower amplitude, depicted by the interleaved (Condition 2). (C) The signal and the masker were delivered to the same electrode at different rates $(1000 \mathrm{~Hz}$ for the signal and $3333 \mathrm{~Hz}$ for the masker) simultaneously (the signal pulse overlapped with every third pulse of the masker; Condition 3). (D) The signal and the masker were delivered to two different electrodes at the same rate, interleaved (Condition 4).

dotted horizontal line in each panel. Condition 1 simultaneously delivered the signal and the masker at the same rate to the same electrode (panel A). The stimulus configuration in Condition 1 could be considered to be the tone-on-tone masking paradigm or the incremental detection in acoustic hearing 
(Bacon and Viemeister 1985). Condition 2 was identical to Condition 1, except that the signal and the masker were interleaved in the first $10 \mathrm{~ms}$, resulting in a 0.5 -ms separation between the signal pulse and masker pulse (panel B). Condition 3 simultaneously delivered the signal and the masker to the same electrode, but at different stimulation rates: $1000 \mathrm{~Hz}$ for the signal and $3000 \mathrm{~Hz}$ for the masker (panel $\mathrm{C}$ ). The stimulus configuration in Condition 3 could be considered a test for the "off-frequency" mechanism in the time domain (Carlyon and White 1992). Condition 4 tested the off-frequency mechanism in the spectral (or place) domain because the $1000-\mathrm{Hz}$ signal and the $1000-\mathrm{Hz}$ masker were delivered to two different electrodes (panel D). In addition, Condition 4 provided a simultaneous masking pattern that could reflect the direction and extent of excitation in electric stimulation, which had been traditionally measured by a forward masking paradigm (Chatterjee and Shannon 1998; Lim et al. 1989).

The bipolar stimulation mode $(\mathrm{BP}+1)$ was used in all conditions with the Nucleus device. Experiments 1-3 presented all stimuli to the same middle electrode pair $(11,13)$. Condition 4 presented the masker to the electrode pair $(11,13)$ and the signal to a different electrode pair from the most basal ( 1 , $3)$ to the most apical $(20,22)$. Figure 2 shows absolute detection thresholds (i.e., no masker) for the 10-ms signal across all $\mathrm{BP}+1$ electrode pairs used in the experiment. These threshold data showed both large intersubject variability $(44-57 \mathrm{~dB})$ and large within-subject interelectrode variability (e.g., 44-54 dB in S2). To overcome this large variability in the absolute threshold, the threshold shift (re: the

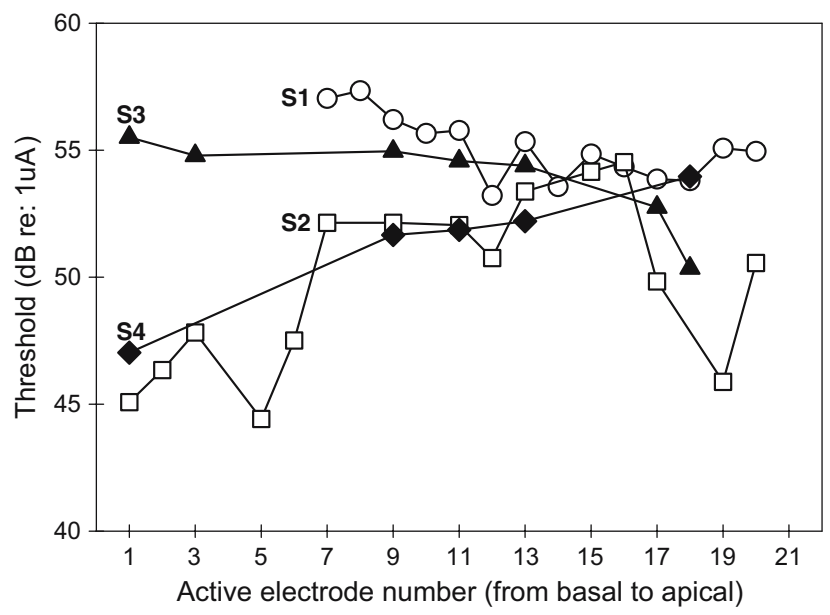

FIG. 2. Absolute threshold ( $\mathrm{dB}$ re: $1 \mu \mathrm{A})$ as a function of electrode number in a bipolar stimulation mode in four Nucleus implant users. The masked threshold all used this absolute threshold as a reference (Figs. 5-8). absolute threshold) was used to facilitate statistical analysis. The 400-ms masker threshold was essentially the same as the 10-ms signal threshold. Unless specified otherwise, the onset condition represented a 0-ms signal delay and the steady-state condition represented a 200-ms signal delay. Three masker levels were used in the experiment, including subthreshold (2 $\mathrm{dB}$ less than the threshold), threshold, and the most comfortable loudness level.

Electric stimuli were delivered to the Ineraid subjects via an optically isolated current source. A foot-paddle safety switch was employed as a release in the unlikely event of overstimulation. A detailed description of the stimulus generation and delivery can be found in previous publications (Zeng and Shannon 1992, 1994). In contrast, electric stimuli were delivered to the Nucleus subjects transcutaneously via a customized research interface, which was previously described in detail (Robert 2002; Shannon et al. 1990).

\section{Procedure}

A three-interval, forced-choice adaptive procedure was used in all experiments (Levitt 1971). The signal was randomly presented in one of the three intervals, whereas the masker was presented in all intervals. The subject had to choose the interval containing the signal. Initially, the signal level was high to allow easy detection of the signal. The signal level was reduced with three consecutive correct responses but increased with any incorrect response. A reversal occurred with either three consecutive correct responses to one incorrect response or vice versa. The step size for the signal level manipulation was large for the first four reversals, but was small afterwards (the exact size depended on the dynamic range of each subject). The detection threshold was estimated by averaging the signal level from the last eight reversals with a small step size, corresponding to the $79 \%$ correct response level. The typical standard deviation for these estimates was about $0.8 \mathrm{~dB}$ for the reported Ineraid data and $1 \mathrm{~dB}$ for the reported Nucleus data.

For statistical analysis, a paired $t$-test was employed to measure whether the overshoot effect reached a significance level in the Ineraid users. An ANOVA with a within-subject, two-way, repeatedmeasures design was employed to measure whether the overshoot effect reach a significance level in the Nucleus users. The factors in the ANOVA included masker level [subthreshold, threshold, and most comfortable loudness (MCL)], and signal delay (onset and steady-state conditions). The significance level was set at $p<0.05$. Should significant interactions occur between the factors in the ANOVA, a 
Temporal edge enhancement in Ineraid implant subject \#1

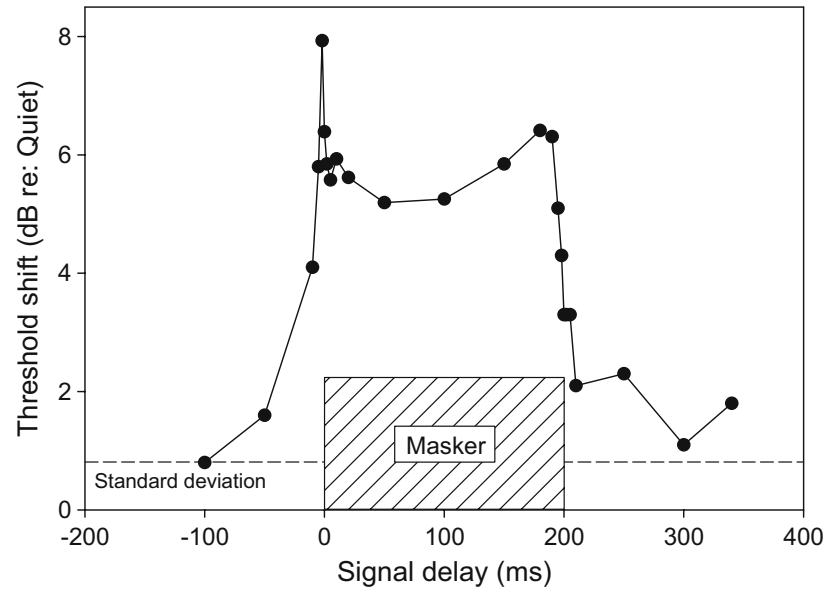

FIG. 3. Temporal edge enhancement measured by threshold shift in Ineraid implant subject \#1. The horizontal dashed line represents 1 standard deviation from the absolute threshold (i.e., $0 \mathrm{~dB}$ on the $y$-axis). The $x$-axis is the signal delay with the negative value representing backward masking, values $0-200 \mathrm{~ms}$ representing simultaneous masking, and values greater than $200 \mathrm{~ms}$ representing forward masking.

one-way ANOVA was performed to reveal leveldependent significant differences.

\section{RESULTS}

\section{Ineraid implant subjects}

Figure 3 shows the threshold shift as a function of signal delay in Ineraid subject \#1. The dashed line represents the signal threshold without the masker plus 1 standard deviation $(35.7+0.8 \mathrm{~dB}$, re: $1 \mu \mathrm{A})$. The shaded area represents the temporal position of the masker with the masker level at $40 \mathrm{~dB}$. Negative signal delays represent backward masking, whereas signal delays greater than $200 \mathrm{~ms}$ represent forward masking. No backward masking was observed at the 100-ms delay but residual forward masking (1-2 dB) was present at the longest delay tested $(150 \mathrm{~ms})$. A 3-dB overshoot effect (with a threshold difference between 0 - and $100-\mathrm{ms}$ delays) was observed in this subject. A 1-dB difference was also observed between the steady-state (100-ms delay) and the offset conditions (190-ms delay).

Figure 4 shows the signal threshold as a function of the masker level in Ineraid subject \#2. The horizontal dashed line represents the signal threshold without the masker $(28.0 \mathrm{~dB}$, re: $1 \mu \mathrm{A})$. The circles represent the signal thresholds obtained with the sinusoidal masker. The inverted triangles represent data obtained with the noise masker. The filled symbols represent thresholds near the onset of the masker (3 ms), whereas the unfilled symbols represent thresholds during the steady-state portion of the masker $(300 \mathrm{~ms})$. A consistent $0.5-2.0 \mathrm{~dB}$ overshoot effect was observed for the sinusoidal masker over the entire masker level range. The 1.4-dB overshoot effect averaged over all masker levels was significant ( $t$-test, $p<0.05)$. No consistent overshoot was observed for the noise masker: The $0.2-\mathrm{dB}$ average overshoot effect was not significant ( $t$-test, $p>0.05)$.

\section{Condition 1. Same-electrode, same-rate,} simultaneous stimulation

Figure 5 shows the averaged threshold shift as a function of the masker level in four Nucleus implant subjects. The circles represent data at the masker onset, whereas the triangles represent data at the masker steady-state position. The same convention will be used to describe data from Exp. 2 and 3. No significant overshoot was observed in terms of the difference in signal threshold shift between the onset and the steady-state conditions $\left(F_{1,3}=0.02, p>0.05\right)$. As expected, a significant effect of the masker level was observed, with the averaged threshold shift being increased from 2.4 to 4.2 when the masker level was increased from subthreshold to threshold, and from 4.2 to $6.9 \mathrm{~dB}$ when the masker level was further increased to MCL $\left(F_{2,6}=19.8, p<0.05\right)$. Finally, no significant interaction was observed between the masker level and the signal delay $\left(F_{1,3}=4.0, p>0.05\right)$.

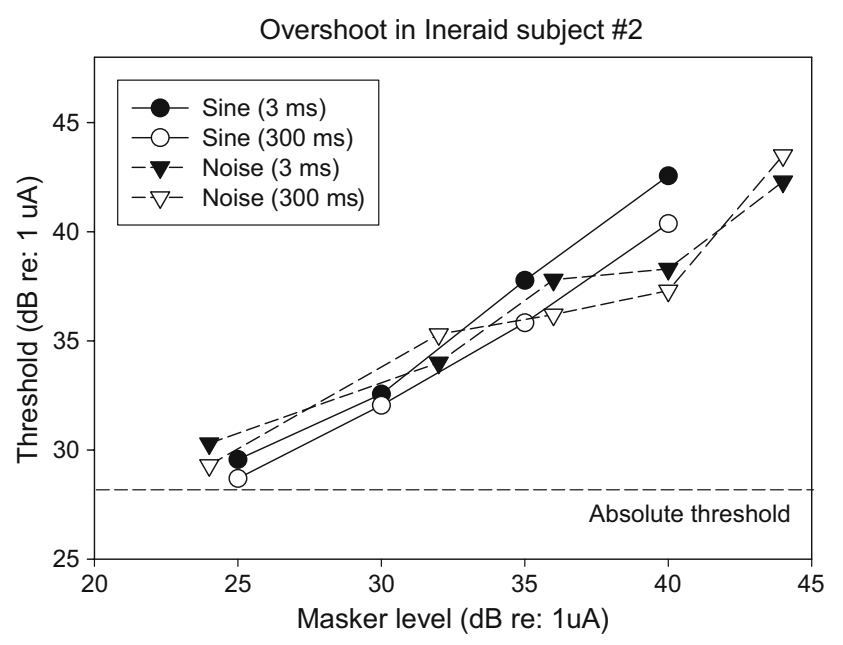

FIG. 4. Signal threshold (y-axis) as a function of masker level (xaxis) in Ineraid subject \#2. Circles represent data collected with the sinusoidal masker. Inverted triangles represent data collected with the noise masker. Filled symbols represent the 3-ms signal delay condition, whereas the unfilled symbols represent the 300-ms signal delay condition. The dashed line represents absolute signal threshold (i.e., no masker). 


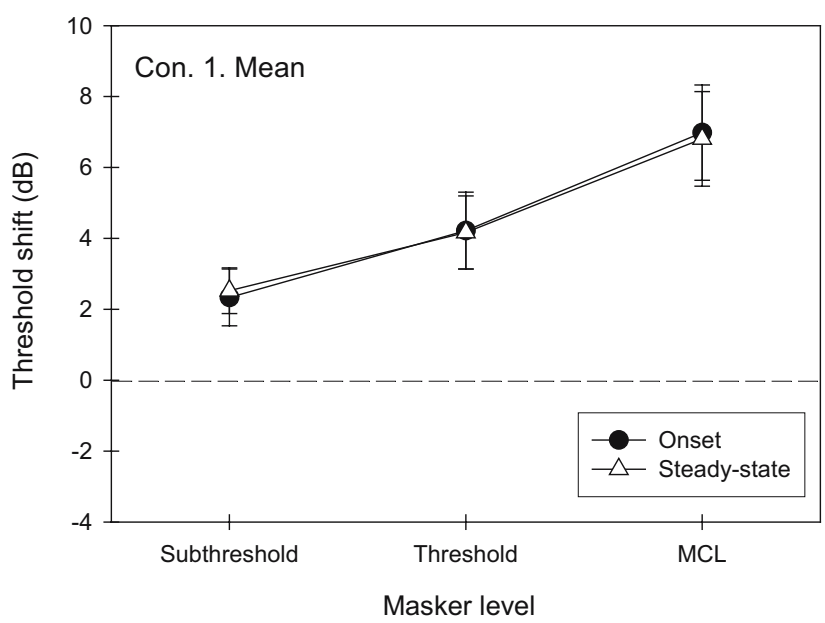

FIG. 5. Average data showing threshold shift ( $y$-axis) as a function of the masker level ( $x$-axis) in Condition 1. Filled circles represent data at the 0-ms signal delay (onset). Unfilled triangles represent data at the 200-ms signal delay (steady state). Error bars represent \pm standard deviation.

Condition 2. Same-electrode, same-rate, interleaved stimulation

Figure 6 shows the average data from the same four subjects in Condition 2, which is significantly different from the pattern of results as observed in Condition 1. First, note a significant interaction between the masker level and the signal delay $\left(F_{2,6}=\right.$ $5.1, p<0.05)$. The significant interaction is evidenced by the crossover pattern in the threshold shift between the low- and high-masker levels. Second, note that the averaged threshold shift was $1.6 \mathrm{~dB}$ higher for the onset than the steady-state condition at the MCL, but was 1.6 and $0.6 \mathrm{~dB}$ lower for the onset condition at subthreshold and threshold masker levels, respectively. The latter was indicative of an "undershoot" effect, namely, the signal was actually more easily detected at the onset than in the steadystate condition. Paired $t$-tests showed that the $1.6-\mathrm{dB}$ overshoot was significant at the MCL and that the 1.6$\mathrm{dB}$ undershoot was significant at the subthreshold level $(p<0.05)$. Third, the negative threshold shift means that the masker actually improved signal detection, producing a signal threshold that was lower than the absolute threshold. Note that the negative threshold shift occurred only under the onset condition at the subthreshold masker level. Finally, similar to the pattern in Condition 1, the effect of masker level was also found to be significant $\left(F_{2,6}=11.3, p<0.05\right)$. The average threshold shift increased from -0.8 to $0.1 \mathrm{~dB}$ when the masker level was increased from the subthreshold to the threshold, and from 0.1 to $4.3 \mathrm{~dB}$ when the masker was further increased to the MCL. These threshold shift values were significantly lower $(2-4 \mathrm{~dB})$ than the comparable values in Condition $1\left(F_{1,3}=41.8 ; p<\right.$ $0.01)$.

Condition 3. Same-electrode, different-rate, simultaneous stimulation

Figure 7 shows the average signal threshold shift from the same four subjects in Condition 3, which appears to have a mixture of the pattern of results between Condition 1 and Condition 2. First, no significant interaction was observed between signal delay and masker level factors $\left(F_{2,6}=4.4, p>0.05\right)$. Second, neither the $-0.8-\mathrm{dB}$ undershoot effect at the subthreshold masker level nor the 0.3-dB overshoot effect at the MCL masker level was significant $\left(F_{1,3}=\right.$ $0.3, p>0.05)$. Third, there was a significant effect of the masker level $\left(F_{2,6}=13.6, p<0.05\right)$, producing an averaged (over both the onset and the steady-state conditions) threshold shift of $0.5,2.5$, and $5.6 \mathrm{~dB}$ at the subthreshold, threshold, and MCL masker levels, respectively. These values fell between the corresponding values in Condition 1 and Condition 2, but were not significantly different from either $(p>$ $0.05)$. Finally, the individual data (not presented) showed that two subjects (S1 and S2) had a negative threshold shift at the subthreshold masker level for the onset signal delay.

\section{Condition 4. Different-electrode, same-rate,} interleaved stimulation

Figure 8 shows the threshold shift as a function of the probe electrode number in the presence of a fixedlevel masker presented to the electrodes $(11,13)$. The electrode number refers to the active electrode

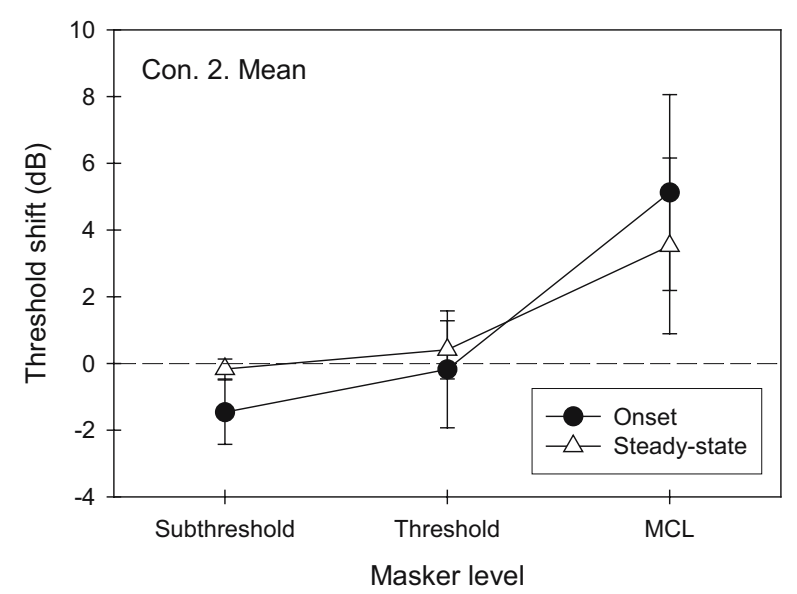

FIG. 6. Average data showing threshold shift ( $y$-axis) as a function of the masker level ( $x$-axis) in Condition 2. Filled circles represent data at the 0-ms signal delay (onset). Unfilled triangles represent data at the 200-ms signal delay (steady state). Error bars represent \pm standard deviation. 


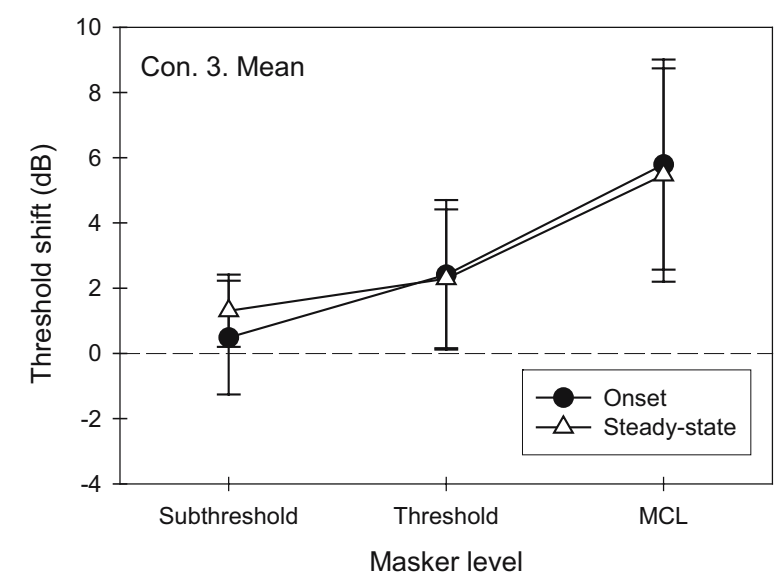

FIG. 7. Average data showing threshold shift (y-axis) as a function of the masker level ( $x$-axis) in Condition 3. Filled circles represent data at the 0-ms signal delay (onset). Unfilled triangles represent data at the 200-ms signal delay (steady state). Error bars represent \pm standard deviation.

number in the $\mathrm{BP}+1$ bipolar configuration mode. The vertical dashed line represents the active electrode number for the masker electrode pair. The triangles, inverted triangles, and circles represent data collected with the masker being presented at the subthreshold, threshold, and MCL, respectively. The filled symbols represent data from the onset condition. The unfilled symbols represent data from the steady-state condition. In subjects S1 and S2, data were not collected at the threshold masker level.

Several interesting results can be noted in the data. First, the overshoot effect was present only at the high masker levels in all four subjects. Second, the overshoot effect appeared to be larger when the signal electrodes were more apical than the masker electrode in all subjects except S2, who had the largest overshoot when the signal and the masker electrodes were the same. Third, there was a clear undershoot effect at low masker levels: The onset condition produced 4-6 dB lower threshold than the steady-state condition in S1 and S2 and 2-3 dB in S3 and S4. Finally, there could be two or more peaks in the masker function ( $\mathrm{S} 1$ and $\mathrm{S} 2$ ). The peaks were more likely to occur on the apical site (S1, S2, and S3) than on the same position as the masker electrode (S4).

Because data were not available for all conditions, statistical analysis was conducted using two masker levels (subthreshold and MCL), two signal delays (onset and steady state), and three electrode positions $(9,11$, and 13). A within-subject, repeatedmeasures ANOVA revealed a significant effect of the masker level $\left(F_{1,3}=36.0, p<0.05\right)$ but no significant main effects of the signal delay or the electrode position $(p>0.05)$. However, there was a significant interaction between the masker level and the signal

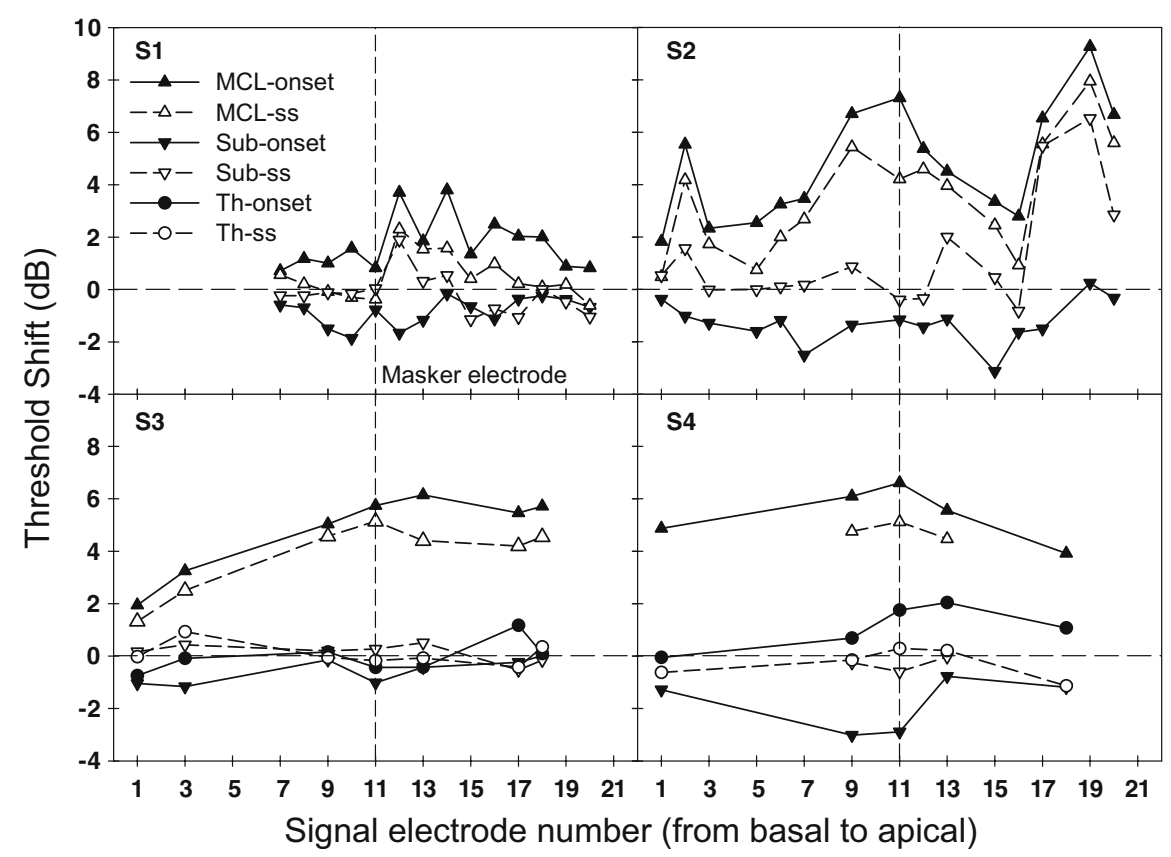

FIG. 8. Individual data showing threshold shift (y-axis) as a function of signal electrode position ( $x$-axis) in Condition 4. The masker was always presented to electrode 11 (vertical dashed line). Triangles, inverted triangles, and circles represent data collected with the masker presented at the most comfortable loudness $(\mathrm{MCL})$ level, the threshold level, and the subthreshold level, respectively. Filled symbols connected by solid line represent data at the 0-ms signal delay (onset). Unfilled symbols connected by dashed line represent data at the 200-ms signal delay (steady state). 
delay $\left(F_{1,3}=16.3, p<0.05\right)$. Collapsing the electrode position factor, post-hoc analysis revealed a significant undershoot effect at the subthreshold masker level (onset threshold - steady threshold $=-1.5 \mathrm{~dB}$, $p<0.05)$ and a significant overshoot effect at the high masker level (onset threshold - steady threshold $=+1.2 \mathrm{~dB}, p<0.05)$.

\section{DISCUSSION}

Overshoot $v s$. undershoot

A set of complicated, yet highly consistent temporal masking patterns in electric hearing was the main finding in the present study. Depending upon the relative difference in stimulus configurations between the signal and the masker, the onset threshold could be higher than (i.e., overshoot), lower than (i.e., undershoot), or the same as (i.e., no effect) the steady-state threshold. Generally speaking, simultaneous stimulation at the same rate and with the same electrode produced no effect of signal delay (Condition 1), whereas interleaved stimulation at different rates or different electrodes showed an effect of signal delay (Conditions 2-4). The masker level was the crucial factor in determining the direction of this signal delay effect. High masker levels produced an overshoot effect, whereas low masker levels produced an undershoot effect.

Although there is a large body of evidence for the presence or absence of the overshoot effect in acoustic hearing, to the best of our knowledge, no one has reported the presence of the undershoot effect. The present results showed that the undershoot effect was always associated with the low-level masker producing an improvement in the signal detection threshold for the onset condition. The low-level masker actually improved the signal detection threshold compared to no masking condition. This improved threshold by a masker is reminiscent of the stochastic resonance effect reported in acoustic and electric hearing, in which a low-level noise also improved the detection threshold of periodic signals (Hong and Rubinstein 2003; Zeng et al. 2000a). In contrast, the present study showed the improved threshold only in the onset condition, whereas other previous studies (Hong and Rubinstein 2003; Zeng et al. 2000a) showed the improved threshold in the steady-state condition (long-duration signal embedded in continuous conditioner or noise). Therefore, the present result suggests a temporal component in stochastic resonance, which has been traditionally modeled as a side effect of the nonlinear system without explicit consideration of the temporal factor (Morse and Evans 1996; Moss et al. 2004; Wiesenfeld and Moss 1995).
Peripheral vs. central mechanisms

The mechanisms underlying the overshoot effect are still unclear, but they most likely have both peripheral and central components. Cochlear nonlinearity and neural adaptation are two peripheral mechanisms that have been linked to the overshoot effect. In electric hearing, the contribution of these two peripheral mechanisms is likely to be minimal, because cochlear nonlinearity is bypassed, whereas neural adaptation is greatly reduced compared with normal neural adaptation in acoustic hearing (Dynes and Delgutte 1992; Javel et al. 1987; Litvak et al. 2001; van den Honert and Stypulkowski 1987). The lack of peripheral contribution might explain the relatively small overshoot effect $(\sim 2 \mathrm{~dB})$ in electric hearing.

The present results suggest a possible central mechanism underlying the observed overshoot effect at the most comfortable loudness only. When all stimulus parameters were kept at the same level between the signal and the masker (i.e., same electrode, same rate, and simultaneous), no overshoot was observed (Condition 1). Whenever one parameter was varied between the signal and the masker (interleaved in Condition 2, different rates in Condition 3, and different electrodes in Condition 4 ), the overshoot or the undershoot effect could be observed. Because the differences in the stimuli were multidimensional, a generic central mechanism, as opposed to a peripheral mechanism, provided a parsimonious explanation for the observed results.

\section{Functional significance}

Overshoot has been suggested to be a biological mechanism to enhance temporal edges (Formby et al. 2000). Previous and present results show interesting interactions of intensive and spectral factors with this temporal edge enhancement. In acoustic hearing, the overshoot effect is maximal at intermediate masker levels. In electric hearing, the overshoot effect occurs only at the most comfortable loudness; additionally, an undershoot effect occurred at low masker levels. At present, our knowledge of temporal edge enhancement is limited, as this enhancement may involve complicated interactions in a multidimensional space. Future studies should investigate what stimulus features are enhanced and where these enhancements occur.

Although the average overshoot or undershoot effect was much smaller in electric hearing $(\sim 2 \mathrm{~dB})$ than in acoustic hearing $(\sim 20 \mathrm{~dB}$ under optimal conditions), its practical significance cannot be ignored. First, one has to view this 2-dB effect in the context of the much narrower dynamic range in 
electric hearing than in acoustic hearing. Compared with the 120-dB acoustic dynamic range, the electric dynamic range is typically limited to $10-20 \mathrm{~dB}$ (Zeng and Galvin 1999; Zeng et al. 2002). Second, the lack of temporal edge enhancement under the same rate and same electrode condition (Condition 1) suggests that current speech processors encoding the temporal envelope with a fixed-rate carrier may not be able to restore dynamic processing in normal acoustic hearing. Several researchers have attempted to enhance temporal envelope representations in electric hearing and achieved some degree of success in terms of improving speech recognition (Geurts and Wouters 1999; Vandali 2001). The amount of enhancement in these studies was arbitrarily determined, lacking a psychophysical basis. The present results show that the amount of enhancement needs to be customized to achieve optimal performance, given the large individual variability of the temporal masking patterns and their delicate dependence on stimulus parameters.

\section{ACKNOWLEDGMENTS}

We thank the cochlear-implant subjects for their help in this study. We thank Abby Copeland, Jay Shah, Jay Rubinstein, and three anonymous reviewers for their helpful comments on an earlier version of the present manuscript. Dr. Shilong Han collected most of the data in the Nucleus subjects when he was a visiting scholar at the Hearing and Speech Research Laboratory, UC Irvine in 2002. This work was supported by the National Institutes of Health 2RO1-DC-002267.

\section{REFERENCES}

Abbas PJ, Sachs MB. Two-tone suppression in auditory-nerve fibers: extension of a stimulus-response relationship. J. Acoust. Soc. Am. 59:112-122, 1976.

Bacon SP. Effect of masker level on overshoot. J. Acoust. Soc. Am. 88:698-702, 1990.

BACON SP, LiU L. Effects of ipsilateral and contralateral precursors on overshoot. J. Acoust. Soc. Am. 108:1811-1818, 2000.

BACON SP, SMith MA. Spectral, intensive, and temporal factors influencing overshoot. Q. J. Exp. Psychol., A 43:373-399, 1991.

BACON SP, TAKAHASHI GA. Overshoot in normal-hearing and hearing-impaired subjects. J. Acoust. Soc. Am. 91:2865-2871, 1992.

BACON SP, Viemeister NF. The temporal course of simultaneous tone-on-tone masking. J. Acoust. Soc. Am. 78:1231-1235, 1985.

Bregman AS. Auditory Scene Analysis: The Perceptual Organization of Sound. The MIT Press, Cambridge, MA, 1994.

Carlyon RP, Sloan EP. The "overshoot" effect and sensory hearing impairment. J. Acoust. Soc. Am. 82:1078-1081, 1987.

Carlyon RP, White LJ. Effect of signal frequency and masker level on the frequency regions responsible for the overshoot effect. J. Acoust. Soc. Am. 91:1034-1041, 1992.
Champlin CA, McFadden D. Reductions in overshoot following intense sound exposures. J. Acoust. Soc. Am. 85:2005-2011, 1989.

Chatterjee M, Shannon RV. Forward masked excitation patterns in multielectrode electrical stimulation. J. Acoust. Soc. Am. 103:2565-2572, 1998.

Chatterjee M, Smith RL. Physiological overshoot and the compound action potential. Hear. Res. 69:45-54, 1993.

Dowling JE. The site of visual adaptation. Science 155:273-279, 1967.

Dynes SB, Delgutte B. Phase-locking of auditory-nerve discharges to sinusoidal electric stimulation of the cochlea. Hear. Res. 58:79-90, 1992.

Elliott LL. Changes in the simultaneous masked threshold of brief tones. J. Acoust. Soc. Am. 38:738-746, 1965.

Fishbach A, Nelken I, Yeshurun Y. Auditory edge detection: a neural model for physiological and psychoacoustical responses to amplitude transients. J. Neurophysiol. 85:2303-2323, 2001.

Formby C, Sherlock LP, Ferguson SH. Enhancements of the edges of temporal masking functions by complex patterns of overshoot and undershoot. J. Acoust. Soc. Am. 107:2169-2187, 2000 .

Geurts L, Wouters J. Enhancing the speech envelope of continuous interleaved sampling processors for cochlear implants. J. Acoust. Soc. Am. 105:2476-2484, 1999.

HaRtLine HK. Visual receptors and retinal interaction. Science 164:270-278, 1969.

Hartline HK, Wagner HG, Ratliff F. Inhibition in the eye of Limulus. J. Gen. Physiol. 39:651-673, 1956.

Hong RS, Rubinstein JT. High-rate conditioning pulse trains in cochlear implants: dynamic range measures with sinusoidal stimuli. J. Acoust. Soc. Am. 114:3327-3342, 2003.

Javel E, Tong YC, Shepherd RK, Clark GM. Responses of cat auditory nerve fibers to biphasic electrical current pulses. Ann. Otol. Rhinol. Laryngol. 96(Suppl. 128):26-30, 1987.

Johnstone BM, YAtes GK. Basilar membrane tuning curves in the guinea pig. J. Acoust. Soc. Am. 55:584-587, 1974.

LevitT H. Transformed up-down methods in psychoacoustics. J. Acoust. Soc. Am. 49:467-477, 1971

Lim HH, TONG YC, CLARK GM. Forward masking patterns produced by intracochlear electrical stimulation of one and two electrode pairs in the human cochlea. J. Acoust. Soc. Am. 86:971-980, 1989.

Litvak L, Delgutte B, Eddington D. Auditory nerve fiber responses to electric stimulation: modulated and unmodulated pulse trains. J. Acoust. Soc. Am. 110:368-379, 2001.

Marr D, Hildreth E. Theory of edge detection. Proc. R. Soc. Lond., B Biol. Sci. 207:187-217, 1980.

MCFAdDen D. Absence of overshoot in a dichotic masking condition. J. Acoust. Soc. Am. 83:1685-1687, 1988.

McFadden D. Spectral differences in the ability of temporal gaps to reset the mechanisms underlying overshoot. J. Acoust. Soc. Am. 85:254-261, 1989

McFadden D, Champlin CA. Reductions in overshoot during aspirin use. J. Acoust. Soc. Am. 87:2634-2642, 1990.

Morse RP, Evans EF. Enhancement of vowel coding for cochlear implants by addition of noise. Nat. Med. 2:928-932, 1996.

Moss F, WARD LM, SANnita WG. Stochastic resonance and sensory information processing: a tutorial and review of application. Clin. Neurophysiol. 115:267-281, 2004.

RHODE WS, Robles L. Evidence from Mossbauer experiments for nonlinear vibration in the cochlea. J. Acoust. Soc. Am. 55:588596, 1974.

Robert ME. House Ear Institute Nucleus Research Interface (HEINRI) Users Guide. House Ear Institute, Los Angeles, CA, 2002. 
Rodieck RW, Stone J. Analysis of receptive fields of cat retinal ganglion cells. J. Neurophysiol. 28:832-849, 1965.

SACHS MB, KIANG NY. Two-tone inhibition in auditory-nerve fibers. J. Acoust. Soc. Am. 43:1120-1128, 1968.

Scharf B, Magnan J, Collet L, Ulmer E, Chays A. On the role of the olivocochlear bundle in hearing: a case study. Hear. Res. 75:1126, 1994.

Scharf B, Magnan J, Chays A. On the role of the olivocochlear bundle in hearing: 16 case studies. Hear. Res. 103:101-122, 1997.

Shannon RV, Adams DD, Ferrel RL, Palumbo RL, Grandgenett M. A computer interface for psychophysical and speech research with the Nucleus cochlear implant. J. Acoust. Soc. Am. 87:905907, 1990.

SMITH RL. Adaptation, saturation, and physiological masking in single auditory-nerve fibers. J. Acoust. Soc. Am. 65:166-178, 1979.

Smith RL, Chatterjee M, Sanpetrino N. The time course of "simultaneous" masking in cochlear implant listeners: an "overshoot" in electrical stimulation? In: Santi PA (ed) Abstracts of the 26th annual midwinter research meeting of the Association for Research in Otolaryngology, p. 806, 2003.

STRICKLAND EA. The relationship between frequency selectivity and overshoot. J. Acoust. Soc. Am. 109:2062-2073, 2001.

Summers V. Overshoot effects using Schroeder-phase harmonic maskers in listeners with normal hearing and with hearing impairment. Hear. Res. 162:1-9, 2001.

VANDALI AE. Emphasis of short-duration acoustic speech cues for cochlear implant users. J. Acoust. Soc. Am. 109:2049-2061, 2001.

van den Honert C, STyPulkowski PH. Temporal response patterns of single auditory nerve fibers elicited by periodic electrical stimuli. Hear. Res. 29:207-222, 1987.

VIEMEISTER NF. Auditory intensity discrimination at high frequencies in the presence of noise. Science 221:1206-1208, 1983.

Wier CC, Green DM, Hafter ER, Burkhardt S. Detection of a tone burst in continuous- and gated-noise maskers; defects of signal frequency, duration, masker level. J. Acoust. Soc. Am. 61:12981300, 1977.

Wiesenfeld K, Moss F. Stochastic resonance and the benefits of noise: from ice ages to crayfish and SQUIDs. Nature 373:33-36, 1995.

WojtcZak M, Donaldson GS, VIEMeISTER NF. Intensity discrimination and increment detection in cochlear-implant users. J. Acoust. Soc. Am. 114:396-407, 2003.

WRIGHT BA. Detectability of simultaneously masked signals as a function of signal bandwidth for different signal delays. J. Acoust. Soc. Am. 98:2493-2503, 1995.

Wright BA, DAI H. Detection of unexpected tones in gated and continuous maskers. J. Acoust. Soc. Am. 95:939-948, 1994a.

WRIGHT BA, DAI H. Detection of unexpected tones with short and long durations. J. Acoust. Soc. Am. 95:931-938, 1994b.

Yost WA. Auditory image perception and analysis: the basis for hearing. Hear. Res. 56:8-18, 1991.

Zeng FG, GaLVIn JJ. Amplitude mapping and phoneme recognition in cochlear implant listeners. Ear Hear. 20:60-74, 1999.

Zeng FG, Shannon RV. Loudness balance between electric and acoustic stimulation. Hear. Res. 60:231-235, 1992.

ZENG FG, SHANNON RV. Loudness-coding mechanisms inferred from electric stimulation of the human auditory system. Science 264:564-566, 1994

Zeng FG, Fu QJ, Morse R. Human hearing enhanced by noise. Brain Res. 869:251-255, 2000a.

Zeng FG, Martino KM, Linthicum FH, Soli SD. Auditory perception in vestibular neurectomy subjects. Hear. Res. 142:102-112, 2000b.

Zeng FG, Grant G, Niparko J, Galvin J, Shannon R, Opie J, Segel P. Speech dynamic range and its effect on cochlear implant performance. J. Acoust. Soc. Am. 111:377-386, 2002.

Zwicker E. Temporal effects in simultaneous masking and loudness. J. Acoust. Soc. Am. 38:132-141, 1965. 